\title{
Paradoxical sleep suppresses immediate early gene expression in the rodent suprachiasmatic nuclei
}

\author{
Michael J. Decker ${ }^{1,2,3}{ }^{*}$, David B. Rye ${ }^{4}$, Shih-Yu Lee ${ }^{1}$ and Kingman P. Stroh/ ${ }^{5}$ \\ 1 Byrdine F. Lewis School of Nursing, Georgia State University, Atlanta, GA, USA \\ 2 Neuroscience Institute, Georgia State University, Atlanta, GA, USA \\ ${ }^{3}$ Center for Behavioral Neuroscience, Georgia State University, Atlanta, GA, USA \\ ${ }^{4}$ Department of Neurology, Emory University, Atlanta, GA, USA \\ ${ }^{5}$ Department of Pulmonary and Critical Care, Veterans Affairs Medical Center, Cleveland, OH, USA
}

\section{Edited by:}

David Gozal, University of Chicago, USA

\section{Reviewed by:}

Hruda N. Mallick, All India Institute of Medical Sciences, India

Debora Hipolide, Universidade Federal de São Paulo, Brazil

\section{${ }^{*}$ Correspondence:}

Michael J. Decker, Georgia State University College of Health and Human Sciences, Urban Life Building, Room 933, , 140 Decatur Street, Atlanta, GA 30303, USA.

e-mail:mdecker@gsu.edu
Light stimulates neuronal activity with subsequent expression of the protein product of the immediate early gene, c-fos, in the Suprachiasmatic Nuclei (SCN). Non-photic stimuli is also thought to modulate activity within the SCN. Here, we sought to determine the effects intrinsic stimuli, specifically, the states of sleep upon c-fos protein expression in the SCN. In 16 rats, c-fos protein expression was evaluated at a fixed time of $1600 \mathrm{~h}$ (subjective night), following $1 \mathrm{~h}$ of electroencephalographically defined sleep. During sleep, as the state of paradoxical sleep (PS) increased, $c$-fos protein expression decreased $(r=-0.41, p<0.033)$. The PS-associated reduction of $c$-fos positive cells occurred equally between animals asleep in the light and those asleep in the dark. We propose a model whereby PS duration might function as a homeostaticentraining mechanism to reduce neuronal activity within the SCN, and thereby modulate circadian rhythms during sleep.

Keywords: suprachiasmatic nucleus, sleep, c-fos

\section{INTRODUCTION}

The Suprachiasmatic Nuclei (SCN) are the neuroanatomic sites of the mammalian biological clock (Miller et al., 1996). Subpopulations of SCN neurons exhibit spontaneous patterns of discharge, and accordingly, are described as self-sustaining neural oscillators, or pacemakers (Welsh et al., 1995; Prosser, 1998; Scheper et al., 1999). When maintained in culture and devoid of afferent input, these neural pacemakers follow a "free-running" discharge pattern lasting 24-25 h (Liu et al., 1997).

Activity of SCN pacemaker cells is significantly influenced by both extrinsic and intrinsic stimuli, which are conveyed through afferent neural pathways. Ambient illumination, the SCN's primary extrinsic stimuli, increases the duration and intensity of neuronal activity with concomitant expression of the protein product of the immediate early gene, $c$-fos (Groos and Meijer, 1985; Rea, 1989; Amir and Robinson, 1995; Meijer et al., 1998). Extrinsic non-photic stimuli also modulate neuronal activity and $c$-fos protein expression within the SCN (Amir and Stewart, 1998). For example, following Pavlonian conditioning techniques in which non-photic and photic stimuli are coupled and presented, nonphotic stimuli alone are able to induce $c$-fos protein expression (Amir and Stewart, 1996).

In addition to extrinsic stimuli, intrinsic stimuli such as behavioral state can modulate activity within the circadian pacemaker. Fear, for example, attenuates light-induced $c$-fos protein expression (Amir and Stewart, 1998; Amir and Stewart, 1999). The state of sleep also influences activity within the SCN (Deboer et al., 2003; Lee et al., 2009).

The SCN itself does not generate the states of wakefulness nor sleep (Mistlberger et al., 1983). Rather, it entrains the appearance of these states to coincide with the diurnal light-dark cycle (Kas and Edgar, 1999). This suggests that the SCN is a primary component within the neuroanatomic circuits modulating the onset and duration of wakefulness and sleep. Therefore, we hypothesized that sleep states might influence patterns of activity within the circadian pacemaker. To evaluate this hypothesis we assessed $c$-fos protein expression within the rodent SCN following electroencephalographically (EEG)-defined wakefulness and sleep, while controlling for the presence and absence of environmental illumination.

\section{MATERIALS AND METHODS STUDY GROUPS}

All protocols were reviewed and approved by the Institutional Animal Care and Use Committee at Case Western Reserve University, where this project was developed and performed. To account for known differences in retinal innervation of the SCN that exist between albino and pigmented rat strains (Steininger et al., 1993), both Sprague Dawley (SD) and Brown Norway (BN) rats were studied. All animals were male, $4-6$ months of age, and were obtained from a single vendor (Harlan). The average weight of the SD was $377 \pm 60$ grams while that of the BN was $294 \pm 35$ grams. While in the animal facility the animals were housed under a 12:12 h light-dark cycle with lights-on beginning at 7:00 AM.

Figure 1 graphically portrays the study protocol. As illustrated, following characterization of sleep/wake architecture, each rat was randomized to one of two separate experimental conditions, then sacrificed and the brain immunohistochemically processed for $c$-fos protein expression. Our preliminary studies suggested that in order to detect any strain-related differences of at least $45 \pm 15 c$-fos positive cells in the SCN (statistical power of $0.90, \alpha=0.05$ ) each of the experimental conditions required four animals from each strain. Consequently, $8 \mathrm{SD}$ and $8 \mathrm{BN}$ rats were used for this study. 


\section{SURGICAL PREPARATION}

Animals were anesthetized with an intraperitoneal injection of pentobarbital sodium $(45 \mathrm{mg} / \mathrm{kg})$. Four holes were drilled at coordinates which would permit recordings of both cortical EEG and hippocampal theta wave activity (Bergmann et al., 1989). One $0.80 \times 3 / 32$ inch stainless steel screw (Vintage Machine, Medina, $\mathrm{OH}, \mathrm{USA}$ ) was placed into each hole and attached to a microconnector (Continental Connector Corp, Trenton, NJ, USA). For the purpose of measuring and recording EMG activity, two wires were inserted into the dorsal neck muscles and also attached to the micro-connector. After placement of the EEG recording screws and EMG fine wire electrodes, the entire matrix was encased in a dental acrylic skullcap that was molded to the shape of the rat's head. The incision around the skullcap was then sutured closed.

\section{PROTOCOL FOR RECORDING SLEEP IN THE LIGHT AND IN THE DARK}

On the seventh day of surgical recovery each animal was acclimatized to a sound attenuated, ventilated, and light-regulated environmental cubicle (BRSLVE Davis, Maryland). The acclimatization protocol consisted of placing the animal into the cubicle $2 \mathrm{~h}$ after light onset (approximately at $0900 \mathrm{~h}$ ), then connecting a cable from a polygraph (Grass model $78 \mathrm{D}$ ) to the micro-connector attached to the animal's head. Inside the cubicle the light intensity was 3,600 lx (General Electric dichroic bulb), the ambient temperature was maintained between $76^{\circ}$ and $79^{\circ} \mathrm{F}$ and food and water were provided ad libitum. With the exception of the small cable attached to the micro-connector mounted on its head, the animal was unrestrained inside of the cubicle.

Each animal was studied on the day following its acclimatization to the environmental cubicle. As illustrated in Figure 1, sleep-wake behavior was characterized between 1130 and $1500 \mathrm{~h}$ on day 8 . Then, at $1500 \mathrm{~h}$, animals were randomly assigned to either remain asleep in the light or else asleep in the dark. Following the conclusion of this hour, at $1600 \mathrm{~h}$, each animal was sacrificed by a lethal intraperitoneal dose of pentobarbital sodium. Each animal was permitted to maintain it's state of wakefulness or sleep up until the moment of injection. Following perfusion (described below), the brain was removed for $c$-fos immunohistochemistry.

\section{TISSUE COLLECTION AND PREPARATION}

Following the lethal dose of pentobarbital sodium, the animal was transcardially perfused with $100 \mathrm{ml}$ of ice-cold heparinized $0.9 \%$ saline solution, followed by $250 \mathrm{ml}$ of $0.4 \%$ paraformaldehyde (PF) in 0.1 molar $(\mathrm{m})$ weight phosphate buffer (PB), followed by $100 \mathrm{ml}$ of $10 \%$ sucrose solution in $0.1 \mathrm{~m} \mathrm{~PB}$. Brains were removed and post-fixed overnight in $4.0 \% \mathrm{PF}$ in $0.1 \mathrm{~m} \mathrm{~PB}$. The following day the brain was placed in a cryoprotectant solution of ethylene glycol and glycerol in $0.4 \mathrm{~m} \mathrm{~PB}$ and stored at $-20^{\circ} \mathrm{C}$.

After all 16 brains had been acquired, they were removed from the freezer, rinsed four times ( $12 \mathrm{~h}$ per rinse) in $0.1 \mathrm{~m} \mathrm{~PB}$ to remove the cryoprotectant. The brains were then soaked in 30\% sucrose solution made in $0.1 \mathrm{~m} \mathrm{~PB}$ until sinking (approximately $48 \mathrm{~h}$ ). Individual brains were placed onto the platform of a freezing microtome and the entire brain sectioned, in the coronal plane, at $50 \mu \mathrm{m}$ per section. All sections from each brain were collected as a 1:4 series and placed into six-well culture dishes containing $0.1 \mathrm{~m}$ tris $\mathrm{PB}$. After all brains had been sectioned, one of the 1:4 series of sections was selected from each rat brain for $c$-fos immunohistochemistry. Individual series of tissue sections were batch-processed in parallel groups of eight brains, in a free-floating fashion, using a plexiglassdivided staining net system (Brain Research).

\section{IMMUNOHISTOCHEMISTRY PROTOCOL}

Endogenous perioxidase activity was eliminated by incubating sections for $60 \mathrm{~min}$ on a rocker platform at room temperature in $100 \mathrm{ml}$ of $0.1 \mathrm{~m}$ tris buffered saline (TBS) with $3.0 \%$ hydrogen peroxide $\left(\mathrm{H}_{2} \mathrm{O}_{2}\right)$ and $1.0 \%$ Triton-X100. Following incubation, sections were rinsed three times in TBS. Sections were then incubated at $+4^{\circ}$ centigrade for $24 \mathrm{~h}$ in a TBS based solution containing $4.0 \%$ goat serum, $0.3 \%$ Triton-X100, and rabbit anti $c$-fos antibody (Cal Biochem) at a 1:10,000 dilution. Following this initial incubation, the sections were rinsed with TBS and then incubated for $2 \mathrm{~h}$ in biotinylated anti-rabbit IgG (Vector Laboratories Inc, Burlingame, CA, USA). Subsequently, sections were again rinsed in TBS and then incubated for $1 \mathrm{~h}$ in an $\mathrm{ABC}$ reagent ( $\mathrm{ABC}$ perioxidase kit, Vector Laboratories Inc, Burlingame, CA, USA). After incubation in the $\mathrm{ABC}$ reagent, tissue sections were rinsed in TBS before a 2- to 5 -min incubation in a filtered TBS solution containing $\mathrm{H}_{2} \mathrm{O}_{2}$, and

\section{Experimental Protocol}

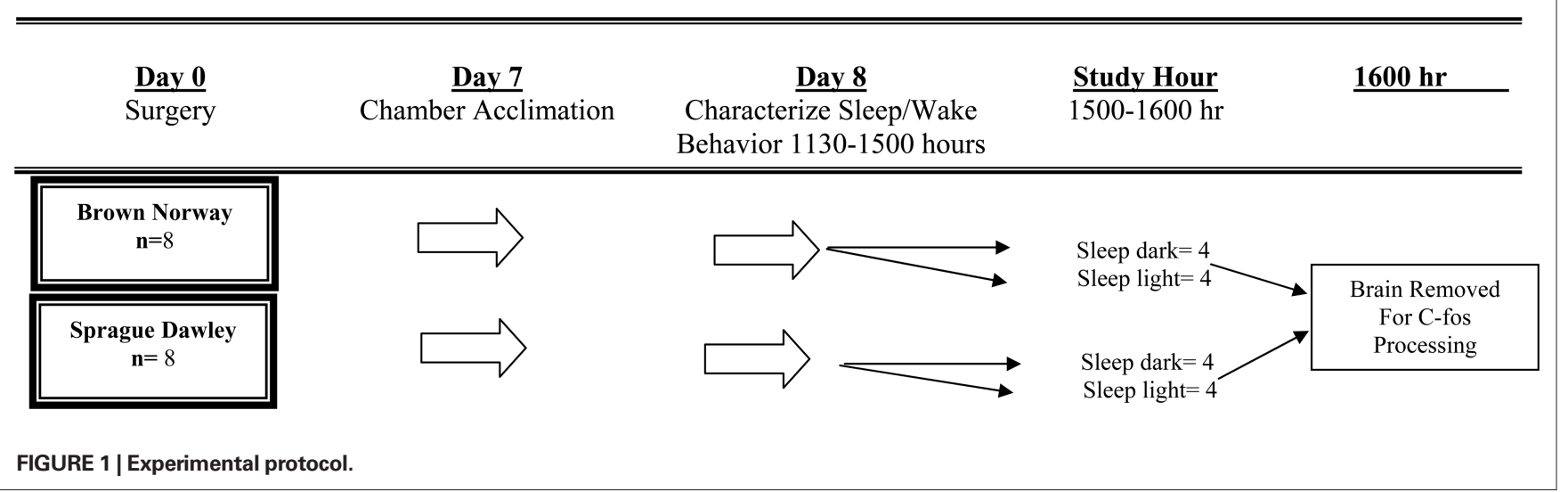


as a chromogen, diaminobenzidine (Sigma) and nickel chloride. Following these steps all sections were mounted on gelatin-coated glass slides, dehydrated, defatted, counter-stained with methyl green and cover-slipped.

\section{DATA ANALYSIS AND STATISTICAL METHODS Scoring of EEG defined wakefulness and sleep}

At the conclusion of the study, each animal's EEG recording was manually scored. Electroencephalographic waveforms were characterized by established frequency and amplitude criteria (Bergmann et al., 1989). Epochs were classified as either awake (W), NREM slowwave sleep (SWS), or paradoxical sleep (PS) (Decker et al., 2003).

\section{Quantification of c-fos positive cells within the SCN}

An Olympus bright-field microscope (20× objective), equipped with a high-resolution color video camera, was used to magnify and project coronal sections of the SCN to a hi-resolution color monitor (Sony Trinitron). To reliably count $c$-fos positive cells within the SCN, it was necessary that the boundaries of the nucleus were welldefined. As a distinct nuclear border does not exist in the most caudal portion of SCN, counting of $c$-fos positive neurons was performed only in the rostral and mid portion of the nucleus. Consequently, two $50 \mu \mathrm{m}$ coronal sections in which the nuclear boundary of the SCN was evident, were selected from each animal. Because the SCN occurs bilaterally, and given hemispheric asymmetries intrinsic to tissue sectioning, this strategy enabled us to count $c$-fos positive cells in four distinct antero-posterior levels per animal. Counting of $c$-fos positive cells was performed by an observer blinded to both the identification of each specimen, as well as the study design. The glass slides containing SCN sections were presented to the scorer in a randomized manner. Cells expressing the $c$-fos protein product were identified as those with a rounded purple-black nucleus.

\section{Statistical approaches to the data}

After each animal's EEG record was scored, values of W, SWS, or PS were entered, epoch by epoch, into a database for statistical evaluation (SPSS, Chicago, IL, USA). The values for numbers of $c$-fos positive neurons from individual SCN cross sections for each animal were also entered into the database. The data were then coded to allow inter- and intra- strain comparisons between the effects of ambient lighting and sleep state architecture with numbers of $c$-fos positive cells within the SCN. The associations between these variables were assessed by bivariate correlation analysis and

Table 1 | Sleep architecture is unaffected by the presence of light during the final hour of sleep.

\begin{tabular}{llll}
\hline SPRAGUE DAWLEY & & & \\
Sleep stage & Lights on & Lights off & 2-tailed significance \\
$\%$ Wake & $12 \% \pm 3 \%$ & $12 \% \pm 5 \%$ & 0.952 \\
$\%$ NREM (NSW + SWS) & $82 \% \pm 3 \%$ & $71 \% \pm 4 \%$ & 0.710 \\
$\%$ PS & $13 \% \pm 8 \%$ & $17 \% \pm 5 \%$ & 0.183 \\
BROWN NORWAY & & & \\
Sleep stage & Lights on & Lights off & 2-tailed significance \\
$\%$ Wake & $6 \% \pm 2 \%$ & $14 \% \pm 4 \%$ & 0.153 \\
$\%$ NREM (NSW + SWS) & $78 \% \pm 1 \%$ & $75 \% \pm 3 \%$ & 0.356 \\
$\%$ PS & $18 \% \pm 2 \%$ & $11 \% \pm 2 \%$ & 0.186
\end{tabular}

relationships between variables explored with $t$-tests, multivariate ANOVA, and linear regression analysis modeling. In all approaches a two tailed significance level of $p<0.05$ was used to determine statistical significance.

\section{RESULTS \\ EFFECTS OF ENVIRONMENTAL ILLUMINATION UPON SLEEP ARCHITECTURE AND $c$-fos EXPRESSION}

During the hour prior to sacrifice (1500-1600), no difference existed between the sleep architecture of either SD or BN rats asleep in the light when compared with those asleep in the dark (Table 1). No strain-related differences existed in values of $c$-fos positive cells in the SCN between SD rats and $\mathrm{BN}$ rats (data not shown) randomized to the same experimental condition (i.e., asleep in the light, asleep in the dark). Therefore, values of sleep-wake architecture and $c$-fos positive cells in the SCN from both SD rats and BN rats were pooled according to experimental condition.

\section{ASSOCIATIONS BETWEEN PARADOXICAL SLEEP AND $c$-fos EXPRESSION IN THE SCN}

Table 2 summarizes correlations between individual sleep stages with $c$-fos protein expression in the SCN. Bivariate analyses reveal a significant inverse correlation between percent of time spent in PS with numbers of $c$-fos positive cells. Figure 2 illustrates that relationship and reveals a significant rsq of $0.41(p=0.033)$. Regression analyses assessing predictors of $c$-fos positive cells in the SCN (percent time awake, percent time in SWS, percent time in PS, presence or absence of light, rat strain) confirm that duration of PS was associated with reduced numbers of $c$-fos positive cells $(p=0.028)$.

Figure 3 demonstrates differences between $c$-fos protein expression within the SCN of two sleeping animals. The rodent sample shown on the left had no PS during the hour prior to sacrifice while the other shown on the right spent $11 \%$ of the hour in PS. That animal, which spent $11 \%$ of the hour in PS, demonstrated a large reduction in $c$-fos positive cells in the ventral portion of the nucleus, a region containing potential pacemakers of the circadian clock (Miller et al., 1996). This pattern of reduction was even more pronounced in those animals which spent a greater amount of time in PS.

\section{DISCUSSION}

We demonstrate that the occurrence and duration of PS is associated with pronounced suppression of $c$-fos protein expression within the SCN. These observations compliment and extend prior studies demonstrating that neuronal firing (Deboer et al., 2003) and gene expression (Lee et al., 2009) within the SCN are also influenced

Table 2 | Bivariate correlations between percent of time spent in each state with numbers of $c$-fos positive cells in the SCN.

\begin{tabular}{llll}
\hline $\begin{array}{l}\text { BN and SD } \\
\text { combined } \boldsymbol{n}=\mathbf{1 6}\end{array}$ & \%Wake & $\begin{array}{l}\text { \%NREM } \\
\text { (NSW + SWS) }\end{array}$ & \% PS \\
\hline $\begin{array}{l}\text { Pearson Correlation } \\
\text { coefficient }\end{array}$ & 0.148 & -0.11 & -0.38 \\
2 tail significance & $0.418 n s$ & $0.536 n s$ & $0.033^{*}$ \\
\hline
\end{tabular}

* Significant at $p<0.05$. 


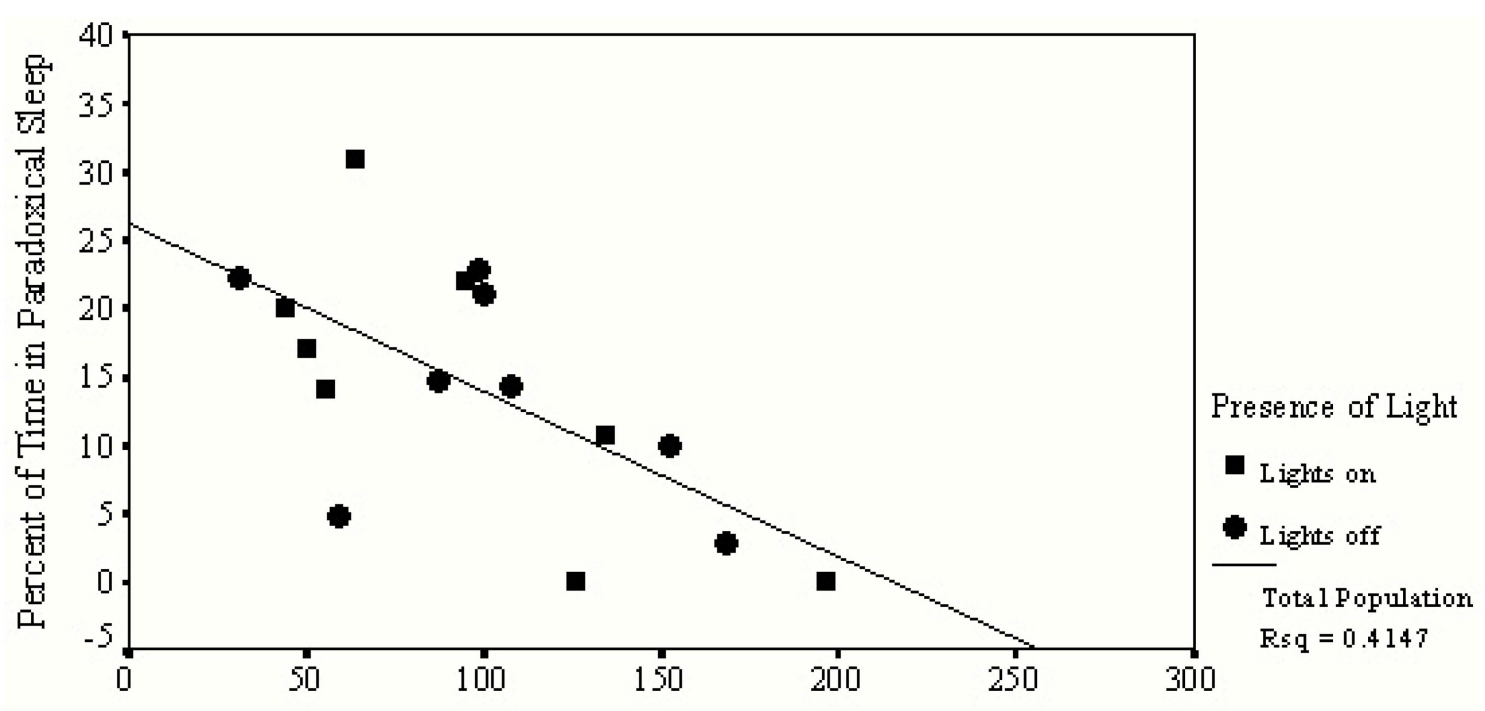

Number of C fos Positive Cells

FIGURE 2 |This figure provides data from only those animals permitted to sleep during the hour prior to sacrifice. Values of $c$-fos positive cells in the SCN of each animal are indicated on the horizontal axis and the amount of time spent in PS is indicated on the vertical axis.
Animals asleep in the light are represented by squares, those asleep in the dark are represented by circles. A significant inverse relationship exists between the number of $c$-fos positive cells found in the SCN and the duration of time spent in PS.

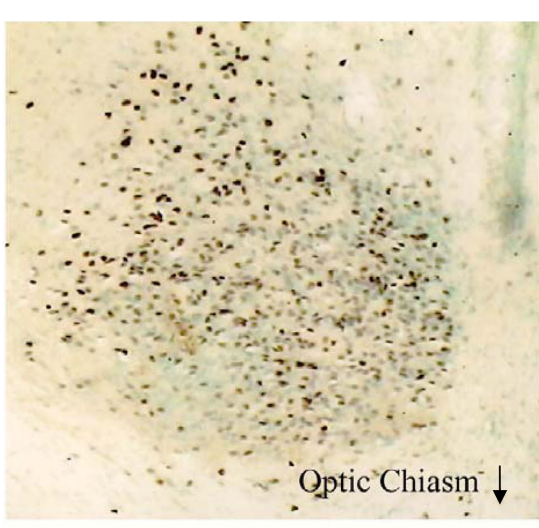

$0 \%$ Paradoxical Sleep

FIGURE 3 |Two photomicrographs of the SCN of two animals asleep in the light during the hour prior to sacrifice. The animal on the left did not have any PS during this time while the animal on the right spent $11 \%$ of the hour in PS. A significant difference in both the number and pattern

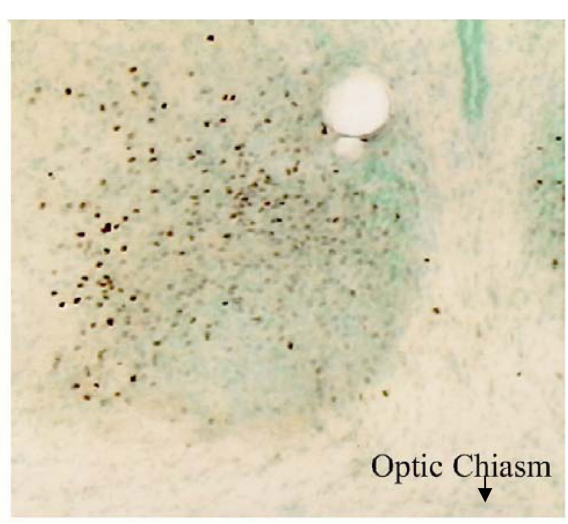

11\% Paradoxical Sleep by the occurrence of PS. In addition, our findings converge with those of Torterolo et al. (2009) who reported (in Table 1 of their publication) that $c$-fos positive cells in the rostral SCN totaled $83.4 \pm 25.6$ during NREM sleep. But, during carbachol-induced REM, that number was reduced to $9.6 \pm 2.7$.

Photomicrographs presented in Figure 3 demonstrate the topographical specificity of PS suppression of $c$-fos protein expression within the SCN. As seen in this figure, the ventral area exhibits the greatest reduction in $c$-fos protein expression following PS. of expression of $c$-fos positive cells (purple dots) can be seen between animals. The most noticeable difference reduction in $c$-fos protein expression occurs in the ventral, retinorecipient portion of the nucleus following PS.
When considering the neural substrates that may contribute to this finding, it is useful to consider those brain regions that: (1) project to and innervate the SCN and; (2) exhibit behavioral state-dependent neural activity, and also; (3) are implicated in behavioral state, particularly PS, control. Those regions and their projections to the SCN are illustrated in Figure 4.

The SCN receives the densest serotonergic input of any hypothalamic nucleus (Bosler and Beaudet, 1985). Release of serotonin into the SCN may be modulated by sleep-wake state to either facilitate or 


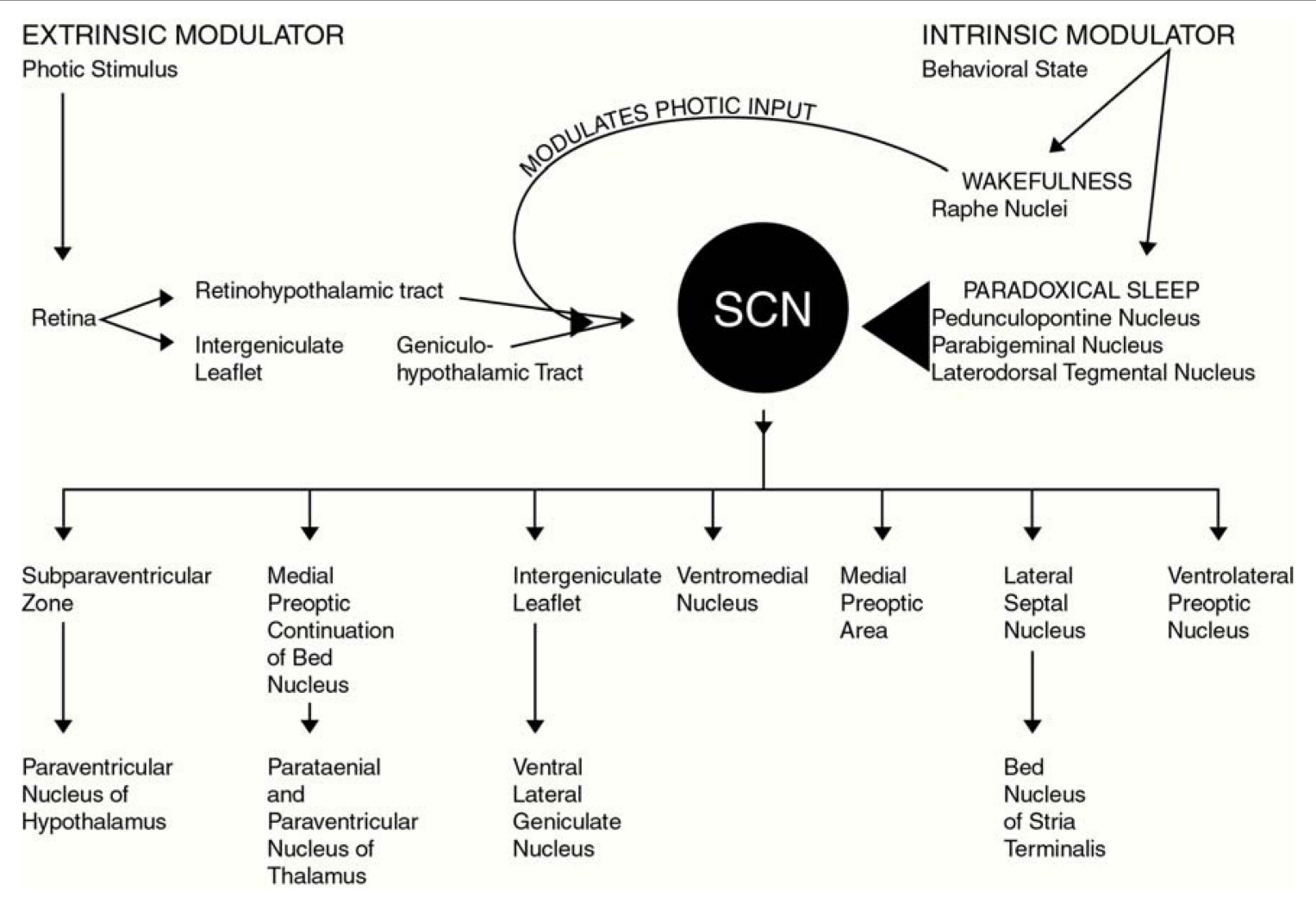

FIGURE 4 |This figure illustrates primary afferent and efferent pathways of the SCN. In addition to the well described retinohypothalamic tract which provides the SCN with extrinsic stimuli (light), the lesser described serotonergic afferent pathway arising from the raphe nuclei, as well as cholinergic afferent pathways originating in the pedunculopontine, parabigeminal and laterodorsal tegmental nuclei (LDT) are also illustrated. This figure graphically illustrates our conceptual model through which intrinsic behavioral state-related stimuli could affect neuronal activity in the SCN. disfacilitate overall neuronal activity levels, and subsequently $c$-fos protein expression (Pickard et al., 1996). A reduction of serotonergic activity that accompanies the onset of PS may lead to a coincident reduction of $c$-fos activity. This mechanism is purely speculative, however, since we did not measure or quantify serotonin activity within the SCN during PS.

Acetylcholine would appear to have a critical role in the PS-specific reduction in $c$-fos protein expression that we observed. Projections originating within the pedunculopontine (PPN) and laterodorsal tegmental nuclei (LDT), which terminate within the SCN (Bina et al., 1993; Rye, 1997) may provide a pathway through which cholinergic inhibition of neuronal activity could occur (Liu et al., 1997) Cholinergic activity in the PPN and LDT increases during PS (Shiromani et al., 1995; Thakkar et al., 1998) and those PPN and LDT neurons involved with the onset and propagation of PS may also be the same which project to the SCN. There, they may potentially suppress neuronal activity and subsequent $c$-fos protein expression. Consistent with this, in vitro SCN slice preparations demonstrate acetylcholine's inhibitory effect on neuronal discharge (Liu and Gillette, 1996). While PS-specific cholinergic inhibition of the circadian pacemaker is only conjectural, it could explain one role of muscarinic receptors in the $\mathrm{SCN}$, particularly since they appear not to participate in transmission of photic stimuli (Takeuchi et al., 1993; Challet et al., 1997).
Our findings further confirm speculation that $c$-fos protein product within the SCN is not only also modulated by extrinsic stimuli such as ambient illumination, but also by intrinsic stimuli, specifically related to PS. Whereas ambient illumination increases neuronal activity and subsequently $c$-fos protein expression, PS appears to dampen neuronal activity and $c$-fos protein expression. Accordingly, we speculate that PS might function as a mechanism to reduce neuronal activity within the SCN, and thereby influence the expression of circadian rhythms (i.e., body temperature and blood pressure (Sano et al., 1995) during sleep. Although plausible substrates through which wakefulness and sleep affect circadian pacemaker do exist (Figure 4), the cellular mechanisms through which this could occur are in need of further elucidation.

\section{LIMITATIONS}

While our findings concur with other those from other investigators who have employed $c$-fos protein expression as a marker of neuronal activity during wakefulness and sleep (Torterolo et al., 2009), this technique is not without procedural and interpretative limitations. First, during immunohistochemical processing, variations in primary, secondary, or tertiary antibody concentrations could lead to differences in staining of $c$-fos positive cells. To minimize potential confounds associated antibody concentrations; immunohistochemical processing was performed by the same 
individual who "batch-processed" groups of eight animal brains to reduce the potential for variations in antibody concentrations and incubation times.

In addition to the immunohistochemical components of this study, potential issues surrounding data analysis and interpretation were also considered. Specifically, to ensure consistency in counting of $c$-fos positive cells, all scoring was performed by the same individual. As an independent measure of quality control, a subset of slides were presented to the scorer on at least two separate occasions, occurring on separate days. Repeat scoring of the same tissue sections typically yielded values within 8.0$10.0 \%$ of previously scored values (data not shown). Finally, an emphasis was placed on selecting sections of SCN with comparable rostral-to-caudal coordinates. This was achieved by noting the presence and shape of the optic chiasm, third ventricle, as well as the shape and size of nearby hypothalamic nuclei such as the paraventricular nucleus.

Despite procedural and interpretative limitations associated with measuring $c$-fos protein expression, we felt that this approach outweighed limitations inherent with techniques such as single cell recordings. We appreciate that measuring the synchronicity between the onset and duration of PS with subsequent $c$-fos expression is not as precise as measuring changes in single cell firing patterns during PS. However, an inherent problem with single cell recordings is adjacent neurons can behave quite differently from the cell being recorded, and those differences will be remain undetected. For example, Lee describes that during the daytime, the clock gene Per1 is active in the ventrolateral portion of the SCN but it is quiescent within the dorsomedial portion of the nucleus (Lee et al., 2009). Single cell recording methodology may not have had the ability to demonstrate those differential levels of activity between neurons within the same discrete nucleus (Lee et al., 2009).

\section{REFERENCES}

Amir, S., and Robinson, B. (1995). Ultraviolet light entrains rodent suprachiasmatic nucleus pacemaker. Neuroscience 69, 1005-1011.

Amir, S., and Stewart, J. (1996). Resetting of the circadian clock by a conditioned stimulus. Nature 379, 542-545.

Amir, S., and Stewart, J. (1998). Induction of Fos expression in the circadian system by unsignaled light is attenuated as a result of previous experience with signaled light: a role for Pavlovian conditioning. Neuroscience. 83, 657-661.

Amir, S., and Stewart, J. (1999). The effectiveness of light on the circadian clock is linked to its emotional value. Neuroscience 88, 339-345.

Bergmann, B. M., Kushida, C. A., Everson, C.A., Gilliland, M.A., Obermeyer, W., and Rechtschaffen, A. (1989). Sleep deprivation in the rat: II. Methodology. Sleep. 12, 5-12.

Bina, K. G., Rusak, B., and Semba, K. (1993). Localization of cholinergic neurons in the forebrain and brainstem that project to the suprachiasmatic

As the resolution between the onset and duration of PS with subsequent $c$-fos protein expression is not exact, we also considered the most appropriate time intervals for our experimental paradigm. In doing so, we chose to employ a protocol similar to that described by Sherin et al. (1996). That ground-breaking publication quantified numbers of $c$-fos positive cells within the ventrolateral preoptic (VLPO) area during wake and sleep, and reported significant differences between those animals that were either awake or asleep during the $60 \mathrm{~min}$ prior to sacrifice. Thus, our rationale to focus on the relationship between $c$-fos expression in the SCN with sleep architecture during the $60 \mathrm{~min}$ prior to sacrifice was based upon Sherin's work and other established protocols (Morgan et al., 1987; Dawes et al., 1996).

Notwithstanding such potential confounds, results here demonstrate that PS is associated with reduced $c$-fos protein expression within the rodent SCN. Our findings extend prior findings by demonstrating that increased durations of PS lead to more pronounced suppression of neuronal activity, as measured by $c$-fos protein expression. Thus, we hypothesize that PS duration might function as a mechanism to reduce neuronal activity within the SCN, and thereby modulate circadian rhythms during sleep. Future studies characterizing cellular mechanisms through which this could occur are needed to define the role of intrinsic stimuli, and their interaction with extrinisic stimuli such as environmental illumination, in maintaining circadian homeostasis.

\section{ACKNOWLEDGMENTS}

We wish to thank Dr. Barbara Freeman of CWRU for her assistance in quantifying $c$-fos protein expression in the SCN, and MaryElizabeth Filon-Prokopius of CWRU and Jeffery Perez of Emory for their assistance with immunohistochemistry. This work is supported in part by the VA Research Service and by the NIH-NHLBI.

Decker, M. J., Hue, G. E., Caudle, W. M., Miller, G. W., Keating, G. L., and Rye, D. B. (2003). Episodic neonatal hypoxia evokes executive dysfunction and regionally specific alterations in markers of dopamine signaling. Neuroscience. 117, 417-425.

Groos, G. A., and Meijer, J. H. (1985) Effects of illumination on suprachiasmatic nucleus electrical discharge. Ann. N. Y. Acad. Sci. 453, 134-146.

Kas, M. J., and Edgar, D. M. (1999). A nonphotic stimulus inverts the diurnalnocturnal phase preference in Octodon degus. J. Neurosci. 19, 328-333.

Lee, M. L., Swanson, B. E., and de la Iglesia, H.O. (2009). Circadian timing of REM sleep is coupled to an oscillator within the dorsomedial suprachiasmatic nucleus. Curr. Biol. 19, 848-852.

Liu, C., Ding, J. M., Faiman, L. E., and Gillette, M. U. (1997). Coupling of muscarinic cholinergic receptors and cGMP in nocturnal regulation of the suprachiasmatic circadian clock. $J$. Neurosci. 17, 659-666.

Liu, C., and Gillette, M. U. (1996). Cholinergic regulation of the suprachiasmatic nucleus circadian rhythm via a muscarinic mechanism at night. J. Neurosci. 16, 744-751.

Meijer, J. H., Watanabe, K., Schaap, J., Albus, H., and Detari, L. (1998). Light responsiveness of the suprachiasmatic nucleus: long-term multiunit and single-unit recordings in freely moving rats. J. Neurosci. 18, 9078-9087.

Miller, J. D., Morin, L. P., Schwartz, W. J., and Moore, R. Y. (1996). New insights into the mammalian circadian clock. Sleep 19, 641-667.

Mistlberger, R. E., Bergmann, B. M. Waldenar, W., and Rechtschaffen, A. (1983). Recovery sleep following sleep deprivation in intact and suprachiasmatic nuclei-lesioned rats. Sleep 6, 217-233.

Morgan, J. L., Cohen, D. R, Hempstead, J. L, and Curran, R. (1987). 4811 mapping patterns of $\mathrm{c}$-fos expression in the central nervous system after seizure. Science 237, 192-197.

Pickard, G. E., Weber, E. T., Scott, P. A., Riberdy, A. F., and Rea, M. A. (1996). 5 HT1B receptor agonists inhibit 
light-induced phase shifts of behavioral circadian rhythms and expression of the immediate-early gene c-fos in the suprachiasmatic nucleus. J. Neurosci. 16, 8208-8220.

Prosser, R. A. (1998). In vitro circadian rhythms of the mammalian suprachiasmatic nuclei: comparison of multi-unit and single-unit neuronal activity recordings. J. Biol. Rhythms 13, 30-38.

Rea, M. A. (1989). Light increases Fosrelated protein immunoreactivity in the rat suprachiasmatic nuclei. Brain Res. Bull. 23, 577-581.

Rye, D. B. (1997). Contributions of the pedunculopontine region to normal and altered REM sleep. Sleep 20, 757-788.

Sano, H., Hayashi, H., Makino, M., Takezawa, H., Hirai, M., Saito, H., and Ebihara, S. (1995). Effects of suprachiasmatic lesions on circadian rhythms of blood pressure, heart rate and locomotor activity in the rat. Jpn. Circ. J. 59, 565-573.

Scheper, T., Klinkenberg, D. Pennartz, C., and van Pelt, J. (1999). A mathematical model for the intracellular circadian rhythm generator. J. Neurosci. 19, 40-47.

Sherin, J. E., Shiromani, P. J., McCarley, R. W., and Saper, C. B. (1996). Activation of ventrolateral preoptic neurons during sleep. Science 271, 216-219.

Shiromani, P. J., Malik, M., Winston, S., and McCarley, R. W. (1995). Time course of Fos-like immunoreactivity associated with cholinergically induced REM sleep. J. Neurosci. 15(5 Pt 1), 3500-3508.

Steininger, T. L., Rye, D. B., Gilliland, M.A., Wainer, B.H., and Benca, R. M. (1993). Differences in the retinohypothalamic tract in albino Lewis versus brown Norway rat strains. Neuroscience 54, 11-14.
Takeuchi, Y., Katoh, Y., and Takahashi, K. (1993). Classical acetylcholine receptors do not play a direct role in neuronal transmission of photic information in the suprachiasmatic nucleus in rats. Neurosci. Lett. 158, 71-74.

Thakkar, M. M., Strecker, R. E., and McCarley, R. W. (1998). Behavioral state control through differential serotonergic inhibition in the mesopontine cholinergic nuclei: a simultaneous unit recording and microdialysis study. $J$. Neurosci. 18, 5490-5497.

Torterolo, P., Benedetto, L., Lagos, P., Sampogna, S., and Chase, M. H. (2009). State-dependent pattern of Fos protein expression in regionallyspecific sites within the preoptic area of the cat. Brain Res. 1267, 44-56.

Welsh, D. K., Logothetis, D. E., Meister, M., and Reppert, S. M. (1995). Individual neurons dissociated from rat suprachiasmatic nucleus express independently phased circadian firing rhythms. Neuron 14, 697-706.

Received: 27 April 2010; paper pending published: 28 May 2010; accepted: 10 August 2010; published online: 22 September 2010.

Citation: Decker MJ, Rye DB, Lee S-Y, and Strohl KP (2010) Paradoxical sleep suppresses immediate early gene expression in the rodent suprachiasmatic nuclei. Front. Neur. 1:122 doi:10.3389/ fneur.2010.00122.

This article was submitted to Frontiers in Sleep and Chronobiology, a specialty of Frontiers in Neurology.

Copyright (c) 2010 Decker, Rye, Lee and Strohl. This is an open-access article subject to an exclusive license agreement between the authors and the Frontiers Research Foundation, which permits unrestricted use, distribution, and reproduction in any medium, provided the original authors and source are credited. 